\title{
Hind Limb
}

National Cancer Institute

\section{Source}

National Cancer Institute. Hind Limb. NCI Thesaurus. Code C77625.

The posterior limb of an animal. 